\title{
Clinical applications of interferon $-\gamma$ releasing assays for cytomegalovirus to differentiate cytomegalovirus disease from bystander activation: a pilot proof-of-concept study
}

Sung-Han Kim ${ }^{1}$,Ho-Su Lee ${ }^{2}$, Hyun-Jung Lee ${ }^{1}$, Sun-Mi Kim', Sung Shin ${ }^{3}$, Sang-Hyoung Park ${ }^{2}$, Kyung-Jo Kim², Young-Hoon Kim³ ${ }^{2}$, Heungsup Sung ${ }^{4}$, Sang-Oh Lee ${ }^{1}$, Sang-Ho Choi ${ }^{1}$, Suk-Kyun Yang ${ }^{2}$, Yang Soo Kim ${ }^{1}$, Jun Hee $\mathrm{Woo}^{1}$, and Duck-Jong $\mathrm{Han}^{3}$

Departments of ${ }^{1}$ Infectious Diseases, ${ }^{2}$ Gastroenterology, ${ }^{3}$ Surgery, and ${ }^{4}$ Laboratory Medicine, Asan Medical Center, University of Ulsan College of Medicine, Seoul, Korea
Received: October 20, 2015 Revised : February 5, 2016 Accepted: February 22, 2016

\section{Correspondence to}

Sung-Han Kim, M.D.

Department of Infectious

Diseases, Asan Medical Center,

University of Ulsan College of

Medicine, 88 Olympic-ro 43-gil,

Songpa-gu, Seoul 05505, Korea

Tel: +82-2-3010-3305

Fax: +82-2-3010-6970

E-mail: kimsunghanmd@hotmail.com Keywords: Cytomegalovirus; Enzyme-linked immunospot assay; Viral load disease were located in the left upper quadrant of the 2-axis model. cases in different types of CMV disease should be proposed.
Background/Aims: We evaluated the proposed clinical application of the combined interpretation of host factors and viral factors in two different cytomegalovirus (CMV) co-infection models.

Methods: We prospectively enrolled all human immunodeficiency virus non-infected patients with confirmed Pneumocystitis jirovecii pneumonia (PCP) and those with suspected gastrointestinal CMV disease in a tertiary hospital. All patients underwent CMV interferon- $\gamma$ releasing assay (IGRA) for CMV (T-track CMV, Lophius Biosciences). We created the 2-axis model with the CMV IGRA results as the $\mathrm{x}$-axis and the results for CMV virus replication as the y-axis, and hypothesized that cases falling in the left upper quadrant (high viral load and low CMVspecific immunity) of the model would be true CMV infections. The CMV IGRA results were concealed from the attending physicians.

Results: Of 39 patients with PCP, four (10\%) were classified as combined CMV pneumonia, 13 (33\%) as bystander activation, and the remaining $22(56 \%)$ as no CMV infection. The data for all four patients with PCP and CMV pneumonia fell in the left upper quadrant of the 2-axis model. Of 24 patients with suspected gastrointestinal CMV disease, 12 (50\%) were classified as gastrointestinal CMV disease and the remaining 12 (50\%) as bystander activation with no gastrointestinal CMV disease. The data for 11 of the 12 patients (92\%) with gastrointestinal CMV

Conclusions: Cases yielding low CMV IGRA results and high CMV viral replication appear to be true CMV infections. Further studies with large number of

\section{INTRODUCTION}

Cytomegalovirus (CMV) is a major cause of morbidity and a preventable cause of mortality in immunocompromised patients [1]. CMV is frequently reactivated in immunocompromised patients, so where there is evi- 
dence of CMV replication it is difficult to differentiate CMV disease from bystander activation [2]. The serologic test of CMV, the detection of CMV viremia (i.e., CMV deoxyribonucleic academia by polymerase chain reaction [PCR], pp65 CMV antigenemia), and virus isolation by CMV culture or PCR from various specimen are the mainstay for the diagnosis of CMV infection. In addition, the direct examination of CMV-specific histopathologic findings with CMV immunohistochemistry or PCR in tissue biopsy is useful in the diagnosis of localized CMV tissue-invasive disease. However, the current diagnostic tests mainly based on viral load testing are suboptimal to diagnose or predict CMV disease differentiated from bystander activation. In the past 20 years, it has become clear that CMV-specific immunity plays a critical role in the development and severity of CMV disease [2]. Since the analysis of CMV-specific $\mathrm{T}$ cell response can potentially allow direct quantification of the patient's ability to control CMV, combining interpretation on host factors and viral factors such as CMV-specific T cell responses and data on CMV replication should help in this difficult clinical situation. Recently, interferon $-\gamma$ releasing assays (IGRAs) for CMV have been developed. Two commercial CMV IGRA have become available on the writing of this manuscript; one is the enzyme-linked immunosorbent assay (ELISA)-based QuantiFERON-CMV (Cellestis, Valencia, CA, USA) and the other, the enzyme-linked immunospot assay (ELISPOT)-based T-track-CMV (Lophius Biosciences, Regensburg, Germany). Theoretically, combining observations on host factors and viral factors such as CMV-specific T cell responses and data on CMV replication should help in this difficult clinical situation. We report here the clinical application of such a combined interpretation in two different CMV co-infection models: (1) confirmed Pneumocystitis jirovecii pneumonia (PCP) patients with suspected CMV pneumonia and (2) patients with suspected gastrointestinal CMV disease.

\section{METHODS}

\section{Study design and definitions}

We prospectively enrolled all non-human immunodeficiency virus (HIV) infected adult patients with confirmed PCP combined with suspected CMV pneumonia, and those with suspected gastrointestinal CMV disease, who were admitted between January 2014 and December 2014 to the Asan Medical Center, a 2,70o-bed tertiary hospital in Seoul, South Korea (Fig. 1). Tests for CMV immunoglobulin G were performed in the hematopoietic stem cell transplant (HCT) recipients and solid organ transplant recipients. All HCT or solid organ transplant recipients were monitored for CMV antigenemia during the post-transplant period. CMV antigenemia of a certain threshold (i.e., > 50 cells per 200,000 cells for kidney transplant and > 1 cells per 200,000 cells for HCT) were indications for preemptive ganciclovir therapy. Microbiological and pathological specimens for diagnosing CMV pneumonia and gastrointestinal CMV disease were processed using standard techniques and procedures, as described previously [3-6]. Decisions regarding anti-CMV therapy, such as ganciclovir, were made by the attending physicians based on each patient's initial clinical features, blood tests, microbiologic results, image findings, and histopathologic findings. To avoid biases, the results of the CMV IGRA were concealed from the attending physicians; this was because the results of the CMV IGRA could have affected decisions on empirical anti-viral therapy. The clinical categories of patients with suspected CMV pneumonia or CMV gastrointestinal (GI) diseases are summarized in

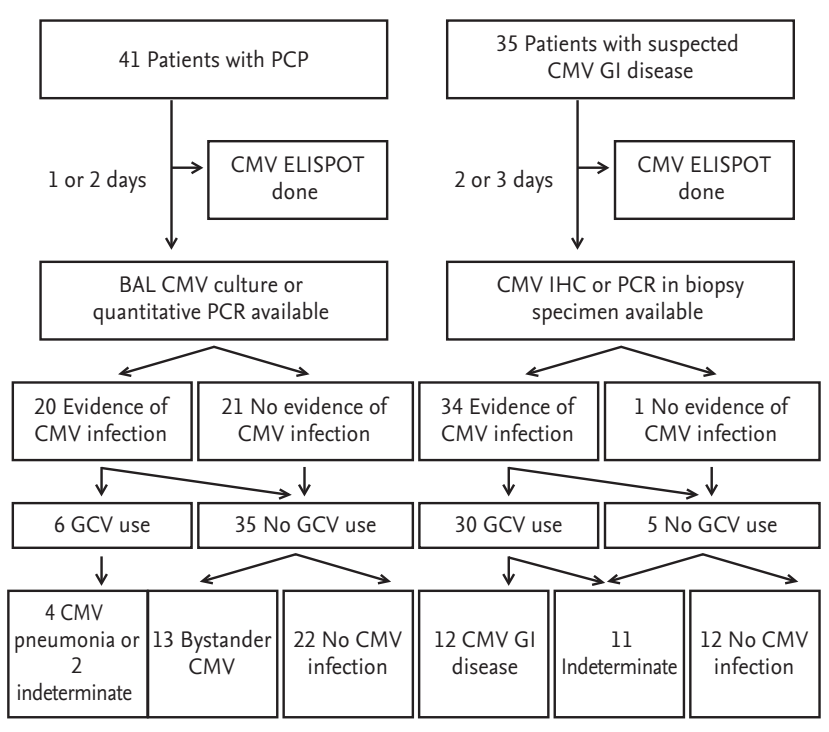

Figure 1. Schematic flow chart. PCP, Pneumocystitis jirovecii pneumonia; CMV, cytomegalovirus; GI, gastrointestinal; ELISPOT, enzyme-linked immunospot assay; BAL, bronchoalveolar lavage; PCR, polymerase chain reaction; IHC, immunohistochemistry; GCV, ganciclovir. 
Table $1[3,4]$. The study protocol was approved by the Institutional Review Board of Asan Medical Center (no. 2014-0198).

\section{Microbiologic methods}

PCP was diagnosed with a positive test result for an immunohistochemical (IHC) antibody assay for P. jirovecii using bronchoalveolar lavage (BAL) fluid in patients with respiratory symptoms and radiologic findings compatible with PCP. All the IHC tests for PCP were read by one experienced medical microbiologist (H.S.). BAL specimens were assayed routinely for CMV infection by shell vial culture. The CMV shell vial consisted of an MRC5 vial inoculated with $0.2 \mathrm{~mL}$ of cell specimen, centrifuged at 1,000 $\times \mathrm{g}$ for 15 minutes, incubated at $35^{\circ} \mathrm{C}$ overnight, and developed with the antibody in a DFA Cytomegalovirus Immediate Early Antigen Identification Kit (Diagnostic Hybrids, Athens, OH, USA). The CMV antigenemia assay using monoclonal antibodies
C10/C11 (Biotest, Dreieich, Germany) was performed as previously described [3-6]. Counts were expressed as positive cells per 200,000 leukocytes. CMV DNA load (CMV quantitative PCR [qPCR]) in EDTA (ethylenediaminetetraacetic acid)-plasma and BAL was measured by qPCR a Cobas Amplicor CMV Monitor-Test Kit on a COBAS Amplicor Analyzer (Roche Molecular Systems, Branchburg, NJ, USA).

\section{ELISPOT}

A peripheral venous blood sample was collected from each patient for the CMV ELISPOT assay for interferon- $\gamma$ producing $\mathrm{T}$ cell responses (i.e., T-track CMV). Briefly, peripheral venous blood $(\sim 8 \mathrm{~mL})$ was obtained and peripheral blood mononuclear cells (PBMC) were immediately (within 30 minutes) separated and collected. The collected cells were suspended at a concentration of $2.0 \times 10^{6}$ cells $/ \mathrm{mL}$, placed $\left(2.0 \times 10^{5}\right.$ cells $/$ well $)$ in 4 wells pre-coated with anti-human interferon- $\gamma$ antibody,

Table 1. Clinical classification of patients with suspected CMV disease

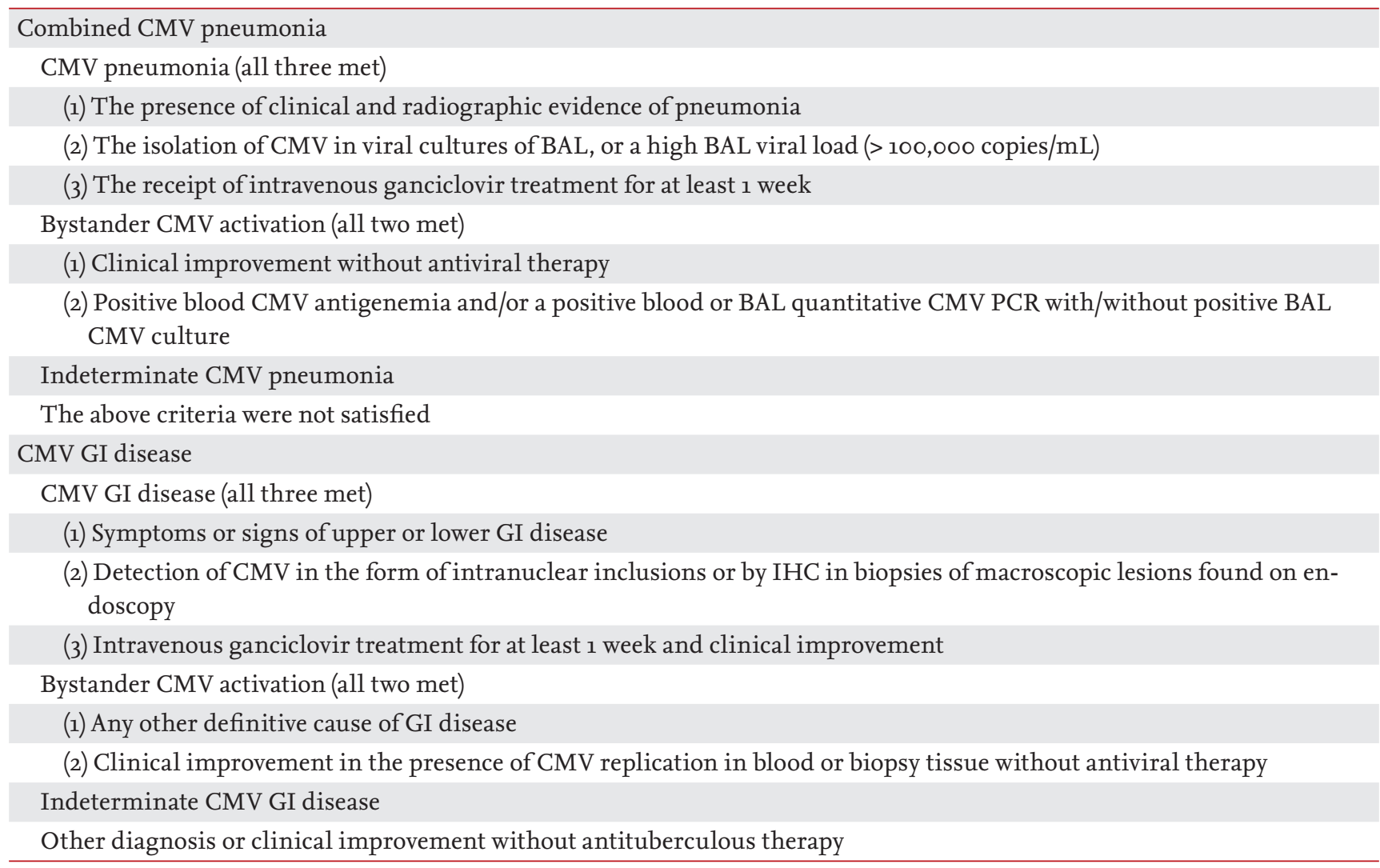

This clinical category is a modified version of that used in the previous studies $[3,4]$.

CMV, cytomegalovirus; BAL, bronchoalveolar lavage; PCR, polymerase chain reaction; GI, gastrointestinal. 
and incubated for 18 hours. As a positive control PHA (Sigma-Aldrich, St. Louise, MO, USA) was added to other wells together with urea-formulated pp65 and urea-formulated IE1 as stimulation antigens, and medium alone served as a negative control, The resulting spots were counted with an automated microscope (ELiSpot $04 \mathrm{HR}$, Autoimmune Diagnostika GmbH, Strassberg, Germany) and the background value, obtained from the negative control wells, was subtracted.

\section{Statistical analysis}

This study is a preliminary proof-of-concept study, so no sample size was calculated. Our hypothesis was that infections with data located in the left upper quadrant (high viral load and low CMV-specific immunity) of the 2-axis model would be true CMV infections. We examined receiving operator characteristic (ROC) curves that plotted sensitivity against the rate of false-positive results over a range of cut-off values. We chose the optimal cut-off value as the point on each ROC curve farthest from the diagonal line that maximized the sum of sensitivity and specificity. Diagnostic performance was expressed in terms of sensitivity, specificity, positive predictive value, and negative predictive value. However, each cut-off value for CMV PCR and CMV IGRA, respectively, in the 2-axis model was selected to maximize the diagnostic performance.

\section{RESULTS}

\section{Patients characteristics}

During the study period, 41 patients with PCP and 35 patients with suspected CMV GI disease were enrolled (Fig. 1). The baseline clinical characteristics of the patients with PCP and with suspected CMV GI disease are shown in Tables 2 and 3, respectively. Of the 41 patients with PCP, four (10\%) were classified as combined CMV pneumonia, $13(32 \%)$ as bystander activation, and 22 (56\%) as no CMV infection. The remaining two (5\%) were classified as indeterminate combined CMV pneumonia and excluded from the final analysis. Of the 35 patients with suspected CMV GI disease, 12 (34\%) were classified as confirmed CMV GI disease and 12 (34\%) as no CMV GI disease with bystander activation. The remaining 11 (31\%) were classified as indeterminate CMV GI disease and excluded from the final analysis. Finally, the 39 patients with PCP and the 24 patients with CMV GI disease and no CMV GI disease were included in our analysis of the proposed model.

\section{2-Axis model in the patients with PCP}

All the 39 patients with PCP were diagnosed on the basis of positive immunohistochemical staining for PCP and received trimethoprim-sulfamethoxazole as primary drug. The detailed microbiologic results for CMV are shown in Table 2 . The sensitivity, specificity, and area under ROC curve of CMV IGRA, BAL CMV qPCR, blood CMV qPCR, and CMV antigenemia for diagnosing combined CMV pneumonia in patients with PCP were shown in Table 4. The area under the ROC curves of pp65-specific T cell response for CMV diseases in patients with PCP was 55 (95\% confidence interval [CI], 39 to 71). Fig. 2 is a dot plot showing the position of each patient in the 2-axis model where the $\mathrm{x}$-axis gives the CMV IGRA results and y-axis the results of the BAL CMV qPCR. It can be seen that the dots representing all four patients with PCP and CMV pneumonia are in the left upper quadrant of the 2-axis model, as predicted. The sensitivity, specificity, positive predictive value, and negative predictive value of high BAL CMV titer (more than $3.5 \mathrm{log}$ ) and low CMV IGRA result (less than 50 spot forming cells (sfc)/200,000 PBMC) for diagnosing combined CMV pneumonia in patients with PCP were $100 \%$ (95\% CI, 4 O to 100), 100\% (95\% CI, 90 to 100), 100\% (95\% CI, 40 to 100 ), and $100 \%$ (95\% CI, 90 to 100), respectively. Other plots where the $\mathrm{x}$-axis gives the CMV IGRA results and the $\mathrm{y}$-axis the results for CMV virus replication as shown by BAL CMV culture, blood CMV qPCR or CMV antigenemia are presented in Supplementary Fig. 1. The diagnostic performance of BAL CMV qPCR and the CMV-specific ELISPOT for predicting 30-day mortality in PCP is shown in Supplementary Table 1.

\section{2-Axis model in the patients with suspected CMV GI disease}

Of the 24 patients with suspected GI CMV disease, 12 were confirmed by positive immunohistochemical staining for CMV and received intravenous ganciclovir as primary drug. The detailed microbiologic results for CMV are shown in Table 3. The area under the ROC curves of pp65-specific T cell response for CMV diseases 
Table 2. Baseline characteristics and outcomes of patients with Pneumocystitis jirovecii pneumonia ${ }^{\mathrm{a}}$

\begin{tabular}{|c|c|c|c|c|c|}
\hline Characteristic & $\operatorname{Total}(\mathrm{n}=41)$ & $\begin{array}{l}\text { Combined CMV } \\
\text { pneumonia }(n=4)\end{array}$ & $\begin{array}{l}\text { Bystander acti- } \\
\text { vation }(n=13)\end{array}$ & $\begin{array}{l}\text { No CMV IFN } \\
\quad(\mathrm{n}=22)\end{array}$ & $\begin{array}{c}\text { Indeterminate CMV } \\
\text { pneumonia }(\mathrm{n}=2)\end{array}$ \\
\hline Age, yr, median (IQR) & $55(35-62)$ & 62 & 57 & 55 & $42(35-48)$ \\
\hline Male sex & $30(73)$ & 4 & $9(69)$ & $15(68)$ & 2 \\
\hline \multicolumn{6}{|l|}{ Underlying diseases } \\
\hline Hematologic malignancy & $18^{c}(44)$ & $3(75)$ & $3(23)$ & $11(50)$ & $1(50)$ \\
\hline Solid organ transplant & $12^{\mathrm{d}}(29)$ & $1(25)$ & $6(46)$ & $5(23)$ & $\mathrm{O}$ \\
\hline Kidney transplant & $8(20)$ & $1(25)$ & $4(31)$ & $3(14)$ & 0 \\
\hline Liver transplant & $3(7)$ & $\mathrm{O}$ & $1(8)$ & $2(9)$ & o \\
\hline Pancreas transplant & $1(2)$ & 0 & $1(8)$ & 0 & 0 \\
\hline Solid tumor & $4(10)$ & 0 & $2(15)$ & $2(9)$ & 0 \\
\hline Steroid user & $2(5)$ & 0 & $1(8)$ & $1(5)$ & 0 \\
\hline HIV infection & $2(5)$ & 0 & o & $1(5)$ & $1(50)$ \\
\hline Others & $3^{e}(7)$ & 0 & $1(8)$ & $2(9)$ & 0 \\
\hline Positive BAL CMV culture & $4(10)$ & $2(50)$ & o & 0 & $2(100)$ \\
\hline Positive BAL CMV qPCR & $12 / 33(36)$ & $4 / 4$ & $7 / 10(70)$ & o/18 & $1 / 1$ \\
\hline Median (IQR) & $3 \cdot 3(0-3 \cdot 0)$ & $4 \cdot 4(3 \cdot 7-5 \cdot 0)$ & $2.9(0-3.2)$ & 0 & 0 \\
\hline Positive blood CMV qPCR & $12 / 31(39)$ & $2 / 4(50)$ & $7 / 8(88)$ & o/17 & $2 / 2$ \\
\hline Median (IQR) & $0(0-2.8)$ & $1.4(0-3 \cdot 5)$ & $2.8(1.3-4.4)$ & 0 & 0 \\
\hline Positive blood CMV antigenemia & $9 / 40(23)$ & $1 / 4(25)$ & $6 / 12(50)$ & $0 / 22$ & $2 / 2$ \\
\hline Median (IQR) & o & $0(0-2.2)$ & $0(0-21.5)$ & o & o \\
\hline $\begin{array}{l}\text { IFN- } \gamma \text { producing } \mathrm{T} \text { cell response, } \\
\text { spot forming cells } / 200,000 \mathrm{PBMC}\end{array}$ & $43 \pm 66$ & $14 \pm 19$ & $39 \pm 63$ & $167 \pm 66$ & NA \\
\hline In-hospital all-cause mortality & $6(15)$ & $2(50)$ & $2(15)$ & $2(9)$ & o \\
\hline
\end{tabular}

Values are presented as number (\%) or mean \pm SD.

CMV, cytomegalovirus; IFN, interferon; IQR, interquartile range; HIV, human immunodeficiency virus; BAL, bronchoalveolar lavage; qPCR, quantitative polymerase chain reaction; PBMC, peripheral blood mononuclear cell; NA, not applicable.

${ }^{a}$ Data are number (\%) of patients with a positive test result/no. of patients tested, unless otherwise indicated.

${ }^{\mathrm{b}}$ No CMV infection was defined if there was no evidence of CMV replication in blood and BAL (negative CMV antigenemia, negative blood CMV PCR, negative BAL CMV PCR, and negative BAL CMV culture).

${ }^{\mathrm{c}}$ Of 18 patients with hematologic malignancies, 6 received hematopoietic stem cell transplant (HCT). Of these 6 HCT recipients, 6 revealed positive CMV immunoglobulin $\mathrm{G}(\mathrm{IgG})$ results before transplant.

${ }^{\mathrm{d}}$ Of 12 patients with solid organ transplant (SOT), 9 underwent CMV IgG tests before SOT. Of 9 SOT recipients, 9 revealed positive CMV IgG results.

${ }^{\mathrm{e}}$ One patient had breast cancer, one interstitial lung disease, and one ulcerative colitis.

in patients with suspected CMV GI disease was 71 (95\% CI, 53 to 85). Fig. 3 is a dot plot for each patient in the 2-axis model where the $\mathrm{x}$-axis is the CMV IGRA results and the y-axis the IHC result for CMV in biopsy tissue. As can be seen, 11 (92\%) of the cases fell, as expected, in the left upper quadrant of the 2-axis model. The sensitivity, specificity, positive predictive value, and negative predictive value of CMV replication in biopsy tissue (positive IHC staining) and low CMV IGRA result (100 sfc/200,000 PBMC) for predicting GI CMV disease in patients with suspected GI CMV disease were 92\% (95\% CI, 62 to 100), 100\% (95\% CI, 74 to 100), 92\% (95\% CI, 62 to 99 ), and $100 \%$ (95\% CI, 73 to 100), respectively. Other models where the $\mathrm{x}$-axis gives the CMV IGRA results and the y-axis the results for CMV viral replication, as shown by CMV PCR in biopsy tissue or blood CMV antigenemia, are presented in Supplementary Fig. 2. 
Table 3. Baseline characteristics and outcomes of patients with suspected gastrointestinal CMV disease ${ }^{\mathrm{a}}$

\begin{tabular}{|c|c|c|c|c|}
\hline Characteristic & Total $(\mathrm{n}=35)$ & $\begin{array}{l}\text { GI CMV disease } \\
\qquad(\mathrm{n}=12)\end{array}$ & $\begin{array}{l}\text { No GI CMV disease }{ }^{\mathrm{b}} \\
(\mathrm{n}=12)\end{array}$ & $\begin{array}{c}\text { Indeterminate CMV } \\
\text { disease }(\mathrm{n}=11)\end{array}$ \\
\hline Age, yr, median (IQR) & $55(43-63)$ & $57(45-61)$ & $49(42-65)$ & $57(35-67)$ \\
\hline Male sex & $17(49)$ & $7(58)$ & $5(46)$ & $5(42)$ \\
\hline \multicolumn{5}{|l|}{ Underlying diseases } \\
\hline Hematologic malignancy & $9^{c}(26)$ & $6(50)$ & $2(17)$ & $1(9)$ \\
\hline Solid organ transplant & $9^{d}(26)$ & $5(42)$ & $3(25)$ & $1(9)$ \\
\hline Kidney transplant & 8 & 4 & 3 & 1 \\
\hline Liver transplant & 1 & 1 & $\mathrm{O}$ & o \\
\hline Inflammatory bowel disease & $14(40)$ & $1(8)$ & $6(50)$ & $7(64)$ \\
\hline Others & $3^{e}(9)$ & o & $1(8)$ & $2(18)$ \\
\hline Positive CMV inclusion body & $18(51)$ & $5(42)$ & $4(33)$ & $9(82)$ \\
\hline Positive CMV IHC & $26(74)$ & $11(92)$ & $5(42)$ & $10(91)$ \\
\hline Positive blood CMV antigenemia & $17(49)$ & $10(83)$ & $1(8)$ & $6(55)$ \\
\hline Median (IQR) & $0(0-24)$ & $29(2-100)$ & o & $1(0-21)$ \\
\hline $\begin{array}{l}\text { IFN- } \gamma \text { producing } \mathrm{T} \text { cell response, } \\
\text { spot forming cells } / 200,000 \mathrm{PBMC}\end{array}$ & $97 \pm 126$ & $19 \pm 25$ & $182 \pm 174$ & $89 \pm 60$ \\
\hline In-hospital all-cause mortality & o & o & o & o \\
\hline
\end{tabular}

Values are presented as number (\%) or mean $\pm \mathrm{SD}$.

CMV, cytomegalovirus; GI, gastrointestinal; IQR, interquartile range; IHC, immunohistochemical; IFN, interferon; PBMC, peripheral blood mononuclear cell.

${ }^{a}$ Data are number (\%) of patients with a positive test result/no. of patients tested, unless otherwise indicated.

${ }^{b}$ No CMV infection was defined if there was no evidence of CMV replication in blood and biopsy tissue (negative CMV antigenemia, negative blood CMV polymerase chain reaction (PCR), negative CMV inclusion body in tissue, negative CMV immunohistochemistry in tissue, and negative CMV PCR in tissue).

${ }^{\mathrm{c}} \mathrm{Of} 9$ patients with hematologic malignancies, 3 received hematopoietic stem cell transplant (HCT). Of these $6 \mathrm{HCT}$ recipients, 3 revealed positive CMV immunoglobulin G (IgG) results before transplant.

${ }^{\mathrm{d}}$ All 9 patients with solid organ transplant (SOT) underwent CMV IgG tests before SOT. Of these 9 SOT recipients, 9 revealed positive CMV IgG results.

'Two patients had no underlying diseases and one IgA nephropathy.

Table 4. Diagnostic performance of each CMV test in patients with Pneumocystis jirovecii pneumonia and suspected CMV pneumonia

\begin{tabular}{lccc}
\hline Variable & Sensitivity $(95 \%$ CI $)$ & Specificity (95\% CI) & AUC (95\% CI) \\
\hline CMV IGRA < 41 sfc/200,000 PBMC & $100(40-100)$ & $32(18-49)$ & $0.55(0.39-0.71)$ \\
BAL qPCR > 2 log & $100(40-100)$ & $75(55-89)$ & $0.94(0.79-0.99)$ \\
Blood qPCR > 2.8 log & $50(7-93)$ & $78(58-91)$ & $0.57(0.39-0.75)$ \\
CMV antigenemia > 2 per 200,000 cells & $25(1-81)$ & $86(71-95)$ & $0.51(0.35-0.67)$ \\
CMV IGRA $(<50$ sfc) and BAL qPCR $>3.5 \log )$ & $100(40-100)$ & $100(90-100)$ & NA \\
\hline
\end{tabular}

CMV, cytomegalovirus; CI, confidence interval; AUC, area under the curve; IGRA, interferon- $\gamma$ releasing assay; sfc, spot forming cell; PBMC, peripheral blood mononuclear cell; BAL, bronchoalveolar lavage; qPCR, quantitative polymerase chain reaction; NA, not applicable. 


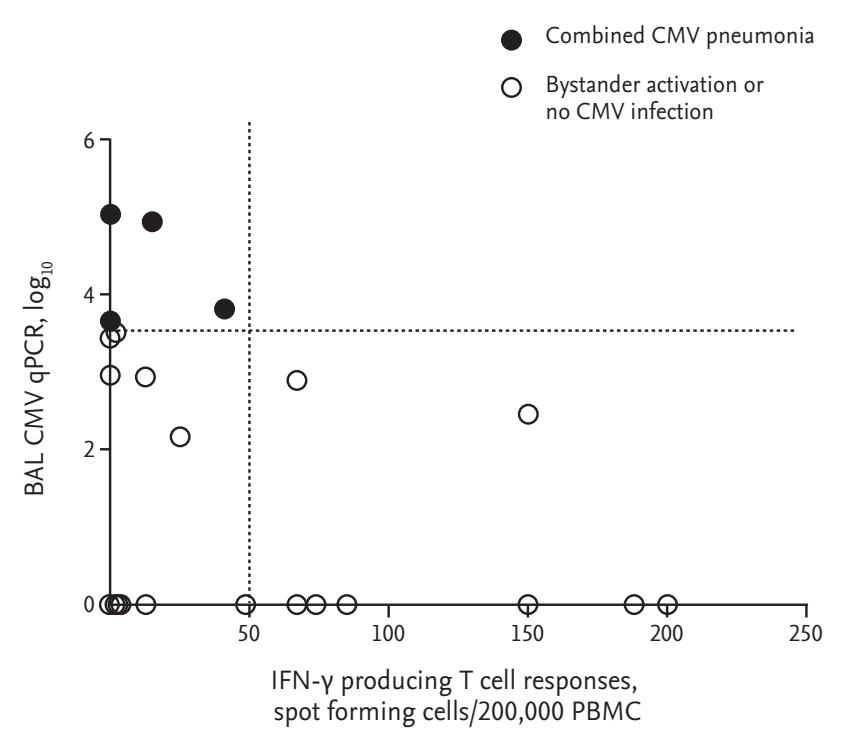

Figure 2. The 2-axis model where the $\mathrm{x}$-axis is the cytomegalovirus (CMV) enzyme-linked immunospot assay result and the $y$-axis the result of bronchoalveolar lavage (BAL) CMV quantitative polymerase chain reaction (qPCR) in patients with Pneumocystitis jirovecii pneumonia. IFN, interferon; PBMC, peripheral blood mononuclear cell.

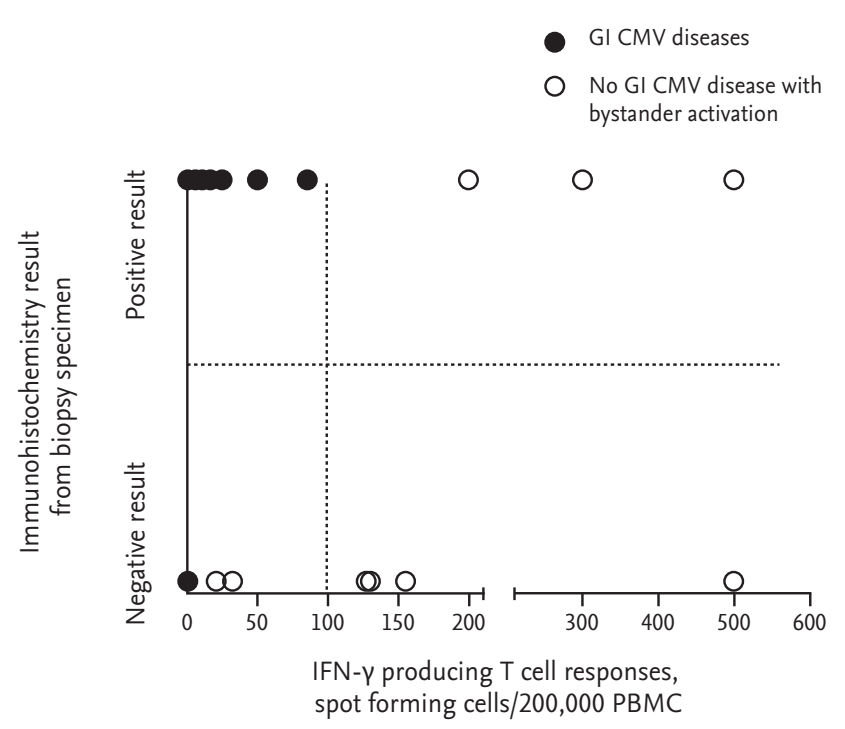

Figure 3. The 2-axis model in which the $\mathrm{x}$-axis is the cytomegalovirus (CMV) enzyme-linked immunospot assay result and the y-axis the result of CMV immunohistochemical staining in patients with suspected gastrointestinal CMV disease. GI, gastrointestinal; IFN, interferon; PBMC, peripheral blood mononuclear cell.

\section{Association between clinical disease category and responses to IEr and pp65}

We investigated whether the responses of the 24 patients

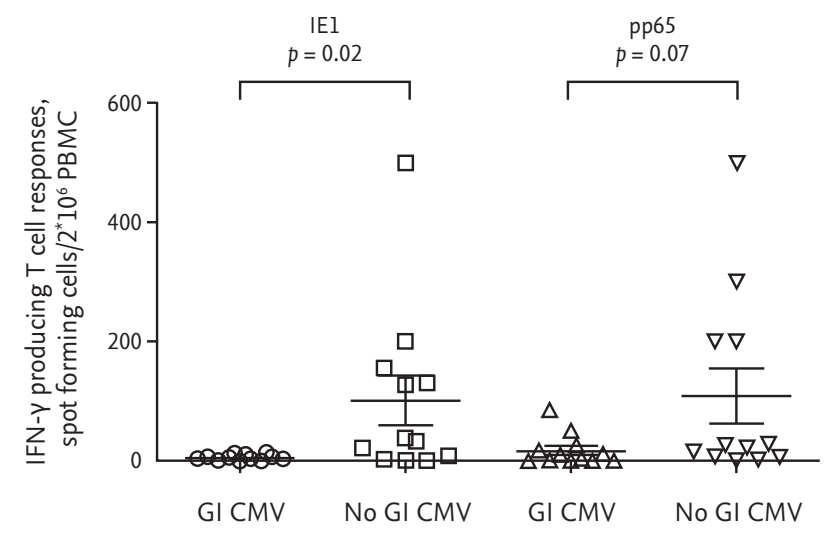

Figure 4. Association between clinical disease category and responses to IE1 and pp65. The responsiveness to IE1 was significantly associated with disease category $(p=0.02)$, while that to pp65 was not significantly associated with disease category $(p=0.07)$. IFN, interferon; PBMC, peripheral blood mononuclear cell; GI, gastrointestinal; CMV, cytomegalovirus.

with suspected GI CMV disease to IE1 and pp65 were associated with their clinical category (Fig. 4). There was a significant association between clinical disease category and responsiveness to IE $1(p=0.02)$, and there was a non-significant indication of an association between responsiveness to pp65 and clinical category $(p=0.07)$.

\section{DISCUSSION}

CMV is frequently reactivated in immunocompromised patients, so when there is evidence of CMV replication it is difficult to differentiate true CMV infection from bystander activation. Until now, diagnosis of CMV disease depended on quantitative or qualitative data on CMV replication in blood, tissue, or urine, together with non-quantitative host factors such as transplant recipient, steroid user, or immunosuppression. However, the presence of a given host risk factor is not merely qualitative, but also a crude measure of host immunologic susceptibility to a certain pathogen such as CMV. The recently developed IGRAs for CMV make it possible to quantify individual immunologic susceptibility to CMV. Theoretically, the combined interpretation of host factors and viral factors such as CMV-specific T cell responses with the data on CMV replication would 
more accurately differentiate CMV disease from bystander activation. This proof-of-concept study showed that the data for true CMV infections fell mostly into the left upper quadrant of the proposed 2-axis model corresponding to infections giving a low CMV-specific cell-mediated immune response along with evidence of (high) virus replication at the local infection site. Hence, our proposed 2-axis model appears to be useful for differentiating true CMV infection from bystander activation.

It has been reported that CMV replication is frequently observed ( $20 \%$ to $70 \%$ positive BAL CMV cultures) in HIV-infected patients with PCP [7]. In general, treatment of the PCP alone often results in clinical improvement without the need to treat the CMV $[8,9]$. Therefore, most of the positive BAL CMV cultures in HIV-infected patients with PCP are regarded as the result of bystander activation. We previously found that about one-thirds of non-HIV patients with confirmed PCP give positive BAL CMV culture results [10]. However, it is largely unknown what proportion of such patients have true coinfections rather than bystander activation in non-HIV patients with PCP. In this study, we differentiated true CMV co-infection from bystander activation according to whether clinical improvement occurred with/without ganciclovir. About half of the patients with PCP (19/41) showed evidence of CMV replication, but only four $(10 \%)$ were classified as CMV pneumonia. Furthermore, our proposed 2-axis model differentiated exactly between true CMV infection and bystander activation. It would therefore be worth investigating whether the proposed 2-axis model also works in HIV-infected patients with PCP. Since the pathophysiologic basis of CMV pneumonia is different in HIV-infected patients and non-HIV patients, different models may be needed to differentiate CMV disease from bystander activation in these two cases. A large prospective study is needed to confirm our conclusion that the combined interpretation of CMVspecific T cell response and CMV replication is of help in diagnosing and treating $\mathrm{CMV}$ pneumonia.

GI CMV disease is a major cause of morbidity and mortality in immunocompromised patients, especially transplant recipients [1] and occasionally causes severe complications in immunocompetent hosts, especially in those of advanced age [11]. The identification of characteristic intranuclear inclusions in stained tissue where the presence of CMV is suspected has been used as the standard reference method for confirming CMV GI disease [4]. However, positive results for immunohistochemical staining or CMV PCR of a biopsy specimen may not be sufficient to diagnose CMV GI disease, although these combined diagnostic method do increase the sensitivity of detection of CMV GI disease. That is because it is difficult to differentiate CMV disease from bystander activation in patients with suspected CMV colitis by using only qualitative evidence of CMV replication at a local site, such as positive IHC or CMV PCR. Our data suggest that the presence of a low CMV-specific $\mathrm{T}$ cell response as part of the evidence for CMV replication at a local site can help in differentiating CMV GI disease from bystander activation in this difficult clinical situation.

Immunosuppressive therapy in inflammatory bowel disease (IBD) frequently induces CMV reactivation in the colonic mucosa, and in turn exacerbates IBD $[12,13]$. It is often difficult to accurately differentiate CMV GI disease from bystander activation in patients with IBD, especially ulcerative colitis [14]. Actually, most patients with IBD with evidence of colonic CMV received ganciclovir therapy in our cohort, as specified in the guidelines of the American College of Gastroenterology [15]. However, the detection of CMV in the colon of a patient with IBD does not necessarily mean GI CMV disease because some patients with CMV detected in their colonic mucosa and not treated with antivirals also go into remission [16]. Therefore, most such cases, if they received ganciclovir therapy, were classified as indeterminate CMV GI disease in this study. Interestingly, high CMVspecific $\mathrm{T}$ cell responses were seen in about half such patients (Supplementary Fig. 2C). It is possible that infections in some patients with high CMV-specific T cell responses resolve spontaneously without use of an antiviral agent, or that they are less resistant to immunosuppressive therapy. In addition, there are promising results showing that CMV GI disease in the inflamed mucosa of patients with ulcerative colitis can be differentiated from bystander activation by quantitative real-time PCR $[16,17]$. Therefore, it would be interesting to evaluate the usefulness for guiding ganciclovir treatment of combined interpretation of quantitative CMV-specific T cell responses and quantitative evidence of CMV replication in the colonic mucosa by differentiating between CMV 
GI disease and bystander activation or predicting resistance to immunosuppressive therapy. Furthermore, the detailed kinetic data about CMV-specific T cell response before and after antiviral therapy will give us more insight on the understanding of pathophysiology of CMV diseases.

Both pp65 and IE1 are considered major T cell targets [13]. A previous study demonstrated that pp65-specific immunity was crucial for controlling the dissemination of CMV in an animal model [18] and that there was a positive correlation between pp65 $\mathrm{T}$ cell responses and CMV viremia [19]. In contrast, others have shown that the level of IE1-speicific T cells correlated with protection from CMV disease in solid organ transplant recipients [17] and was useful for predicting the post transplant risk of CMV infection in kidney transplant recipients [20]. Therefore, our findings of higher levels if IE1-specific T cells than pp65-specific T cell in association with no GI CMV disease is consistent with these previous studies $[17,20]$.

This study has several limitations. First, it is a proofof-concept study and the sample size was not enough to calculate the diagnostic performance of the 2-axis model for CMV disease. Second, there is no established gold standard test for the diagnosis of CMV disease; hence our cases could not be firmly classified as CMV disease. To avoid biases, the results of the CMV IGRA were concealed from the attending physicians because the results of the CMV IGRA could have affected decisions on empirical anti-viral therapy. The classification of bystander activation was relatively clear because clinical improvement without antiviral treatment could be convincingly classified as due to it. However, some patients in which bystander activation actually occurred may have been classified as CMV disease or indeterminate CMV disease if they received antiviral treatment. Finally, our study was not appropriately designed to evaluate the diagnostic performance of CMV quantitative PCR because this test was integrated in the diagnostic criteria. So, the cautious interpretation needed because of a bias favoring diagnostic performance of CMV quantitative PCR.

In conclusion, this study suggests that infections with low CMV IGRA results and high levels of CMV virus replication are true CMV infections. This concept might help clinicians make decisions about antiviral therapy, so further studies with large number of cases in differ- ent types of CMV diseases should be proposed.

\section{KEY MESSAGE}

1. Cytomegalovirus (CMV) is frequently reactivated in immunocompromised patients, so it is difficult to differentiate true CMV infection from bystander activation when there is evidence of CMV replication.

2. We created the 2-axis model with the CMV interferon- $\gamma$ releasing assay results as the $\mathrm{x}$-axis and the results for CMV virus replication as the $\mathrm{y}$-axis.

3. Cases falling in the left upper quadrant (high viral load and low CMV-specific immunity) of the model appear to be true CMV infections.

\section{Conflict of interest}

No potential conflict of interest relevant to this article was reported.

\section{Acknowledgments}

This study was supported by Cheongnam study grant from the Korean Society of Internal Medicine (grant 2014).

\section{REFERENCES}

1. Ramanan P, Razonable RR. Cytomegalovirus infections in solid organ transplantation: a review. Infect Chemother 2013;45:260-271.

2. Egli A, Humar A, Kumar D. State-of-the-art monitoring of cytomegalovirus-specific cell-mediated immunity after organ transplant: a primer for the clinician. Clin Infect Dis 2012;55:1678-1689.

3. Moon SM, Sung H, Kim MN, et al. Diagnostic yield of the cytomegalovirus (CMV) antigenemia assay and clinical features in solid organ transplant recipients and hematopoietic stem cell transplant recipients with CMV pneumonia. Transpl Infect Dis 2012;14:192-197.

4. Jang EY, Park SY, Lee EJ, et al. Diagnostic performance of the cytomegalovirus (CMV) antigenemia assay in patients with CMV gastrointestinal disease. Clin Infect Dis 2009;48:e121-e124. 
5. Park SY, Lee SO, Choi SH, et al. Efficacy and safety of low-dose ganciclovir preemptive therapy in allogeneic haematopoietic stem cell transplant recipients compared with conventional-dose ganciclovir: a prospective observational study. J Antimicrob Chemother 2012;67:14861492.

6. Park SY, Lee SO, Choi SH, et al. Paradoxical rising cytomegalovirus antigenemia during preemptive ganciclovir therapy in hematopoietic stem cell transplant recipients: incidence, risk factors, and clinical outcomes. J Clin Microbiol 2011;49:4179-4184.

7. Millar AB, Patou G, Miller RF, et al. Cytomegalovirus in the lungs of patients with AIDS: respiratory pathogen or passenger? Am Rev Respir Dis 1990;141:1474-1477.

8. Dolin R. AIDS therapy. In: Huang L, Masur H. Pneumocystitis Pneumonia. 3rd ed. Philadelphia: Churchill Livingstone, 2008:637-658.

9. Miles PR, Baughman RP, Linnemann CC Jr. Cytomegalovirus in the bronchoalveolar lavage fluid of patients with AIDS. Chest 1990;97:1072-1076.

10. Kim T, Moon SM, Sung H, et al. Outcomes of non-HIV-infected patients with Pneumocystis pneumonia and concomitant pulmonary cytomegalovirus infection. Scand J Infect Dis 2012;44:670-677.

11. Galiatsatos P, Shrier I, Lamoureux E, Szilagyi A. Meta-analysis of outcome of cytomegalovirus colitis in immunocompetent hosts. Dig Dis Sci 2005;50:609-616.

12. Roblin X, Pillet S, Oussalah A, et al. Cytomegalovirus load in inflamed intestinal tissue is predictive of resistance to immunosuppressive therapy in ulcerative colitis. Am J Gastroenterol 2011;106:2001-2008.

13. Matsuoka K, Iwao Y, Mori T, et al. Cytomegalovirus is frequently reactivated and disappears without antiviral agents in ulcerative colitis patients. Am J Gastroenterol 2007;102:331-337.

14. Lawlor G, Moss AC. Cytomegalovirus in inflammatory bowel disease: pathogen or innocent bystander? Inflamm Bowel Dis 2010;16:1620-1627.

15. Kornbluth A, Sachar DB; Practice Parameters Committee of the American College of Gastroenterology. Ulcerative colitis practice guidelines in adults (update): American College of Gastroenterology, Practice Parameters Committee. Am J Gastroenterol 2004;99:1371-1385.

16. Yoshino T, Nakase $\mathrm{H}$, Ueno $\mathrm{S}$, et al. Usefulness of quantitative real-time PCR assay for early detection of cytomegalovirus infection in patients with ulcerative colitis refractory to immunosuppressive therapies. Inflamm Bowel Dis 2007;13:1516-1521.

17. Bunde T, Kirchner A, Hoffmeister B, et al. Protection from cytomegalovirus after transplantation is correlated with immediate early 1-specific CD8 T cells. J Exp Med 2005;201:1031-1036.

18. Malouli D, Hansen SG, Nakayasu ES, et al. Cytomegalovirus pp65 limits dissemination but is dispensable for persistence. J Clin Invest 2014;124:1928-1944.

19. Pipeling MR, John ER, Orens JB, Lechtzin N, McDyer JF. Primary cytomegalovirus phosphoprotein 65-specific CD8+ T-cell responses and T-bet levels predict immune control during early chronic infection in lung transplant recipients. J Infect Dis 2011;204:1663-1671.

20. Bestard O, Lucia M, Crespo E, et al. Pretransplant immediately early-1-specific $\mathrm{T}$ cell responses provide protection for CMV infection after kidney transplantation. Am J Transplant 2013;13:1793-1805. 
Supplementary Table 1. Diagnostic performance of BAL CMV qPCR and the CMV-specific ELISPOT for predicting 30 -day mortality in PCP

\begin{tabular}{|c|c|c|c|c|c|c|}
\hline \multirow{2}{*}{ Variable } & \multirow{2}{*}{ Sensitivity } & \multirow{2}{*}{ Specificity } & \multicolumn{2}{|c|}{ Predictive value } & \multicolumn{2}{|c|}{ Likelihood ratio } \\
\hline & & & Positive & Negative & Positive & Negative \\
\hline BAL CMV qPCR > 2.52 log copies/mL & $75(35-97)$ & $62(49-73)$ & $19(7-36)$ & $95(85-99)$ & $1.96(1.19-3.24)$ & $0.40(0.12-1.36)$ \\
\hline $\begin{array}{l}\text { CMV-specific ELISPOT }<5 \text { sfu/200,000 } \\
\text { cells }\end{array}$ & $75(35-97)$ & $66(54-77)$ & $21(8-40)$ & $96(85-99)$ & $2.22(1.32-3.73)$ & $0.38(0.11-1.27)$ \\
\hline $\begin{array}{l}\text { BAL CMV qPCR }>2.52 \text { log copies } / \mathrm{mL} \& \\
\text { CMV-specific ELISPOT }<5 \mathrm{sfu} / 200,000 \text { cells }\end{array}$ & $50(16-84)$ & $85(74-93)$ & $29(8-58)$ & $94(84-98)$ & $3.40(1.38-8.35)$ & $0.59(0.29-1.18)$ \\
\hline $\begin{array}{l}\text { BAL CMV qPCR }>2.52 \text { log copies/mL or } \\
\text { CMV-specific ELISPOT }<5 \mathrm{sfu} / 200,000 \text { cells }\end{array}$ & $100(63-100)$ & $43(31-55)$ & $17(8-31)$ & $100(88-100)$ & $1.74(1.42-2.14)$ & - \\
\hline
\end{tabular}

Values are presented as percentage ( $95 \%$ confidence interval), except likelihood ratio. The optimal cut-off point were determined by constructing a receiver operating characteristic (ROC) curve.

BAL, bronchoalveolar lavage; CMV, cytomegalovirus; qPCR, quantitative polymerase chain reaction; ELISPOT, enzyme-linked immunospot assay; PCP, Pneumocystitis jirovecii pneumonia. 
Combined CMV pneumonia

O Incocent bystander or

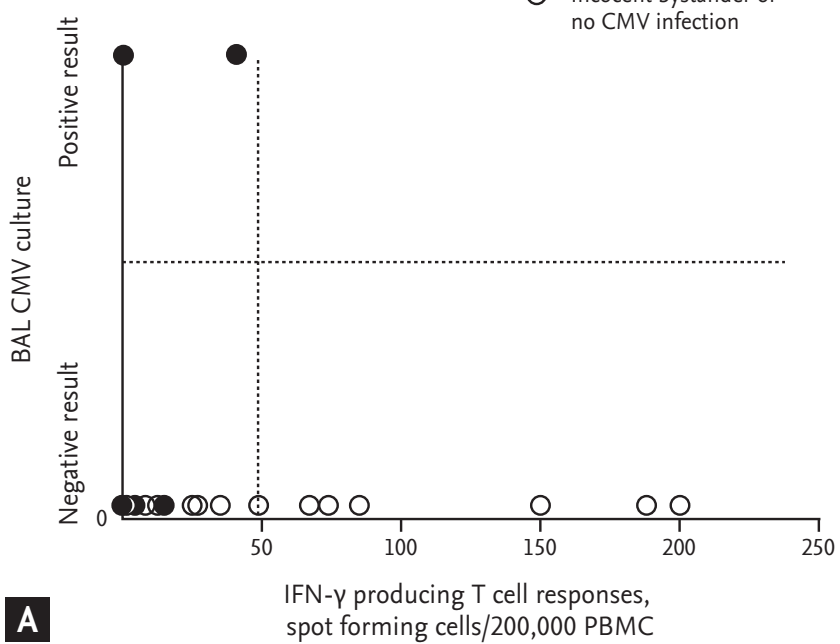

A spot forming cells/200,000 PBMC

- Combined CMV pneumonia

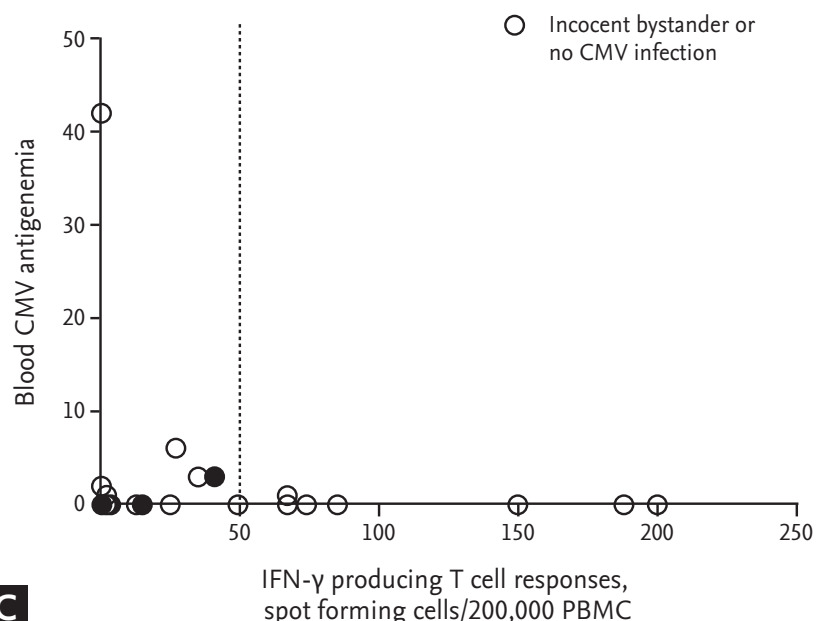

C

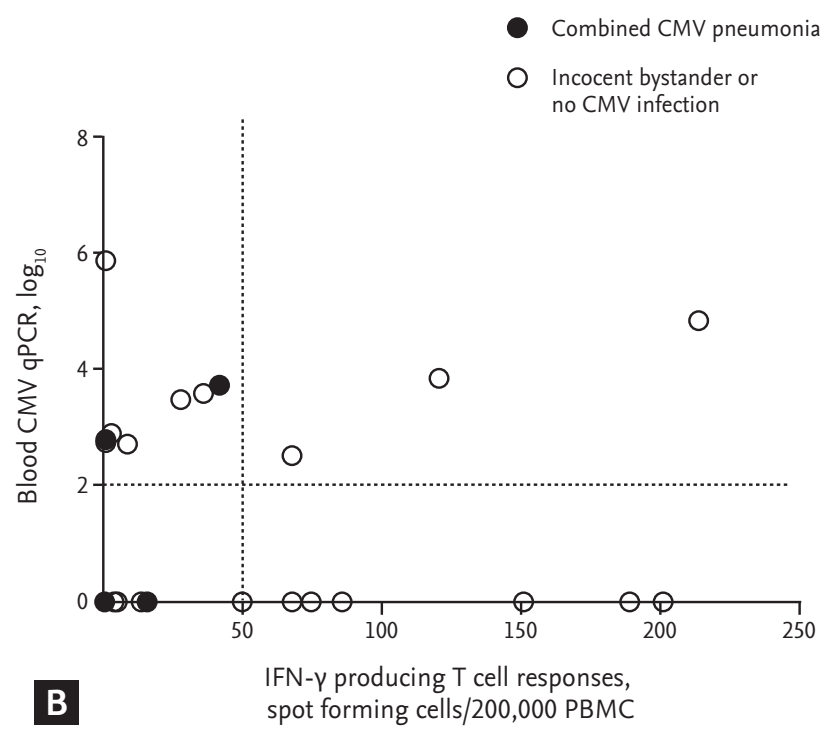

Supplementary Figure 1. The 2-axis model in which the $\mathrm{x}$-axis is the cytomegalovirus (CMV) enzyme-linked immunospot assay result and the y-axis result of bronchoalveolar lavage (BAL) CMV culture (A), blood CMV quantitative polymerase chain reaction (qPCR) (B), and blood CMV antigenemia (C) in patients with Pneumocystitis jirovecii pneumonia. IFN, interferon; PBMC, peripheral blood mononuclear cell. 


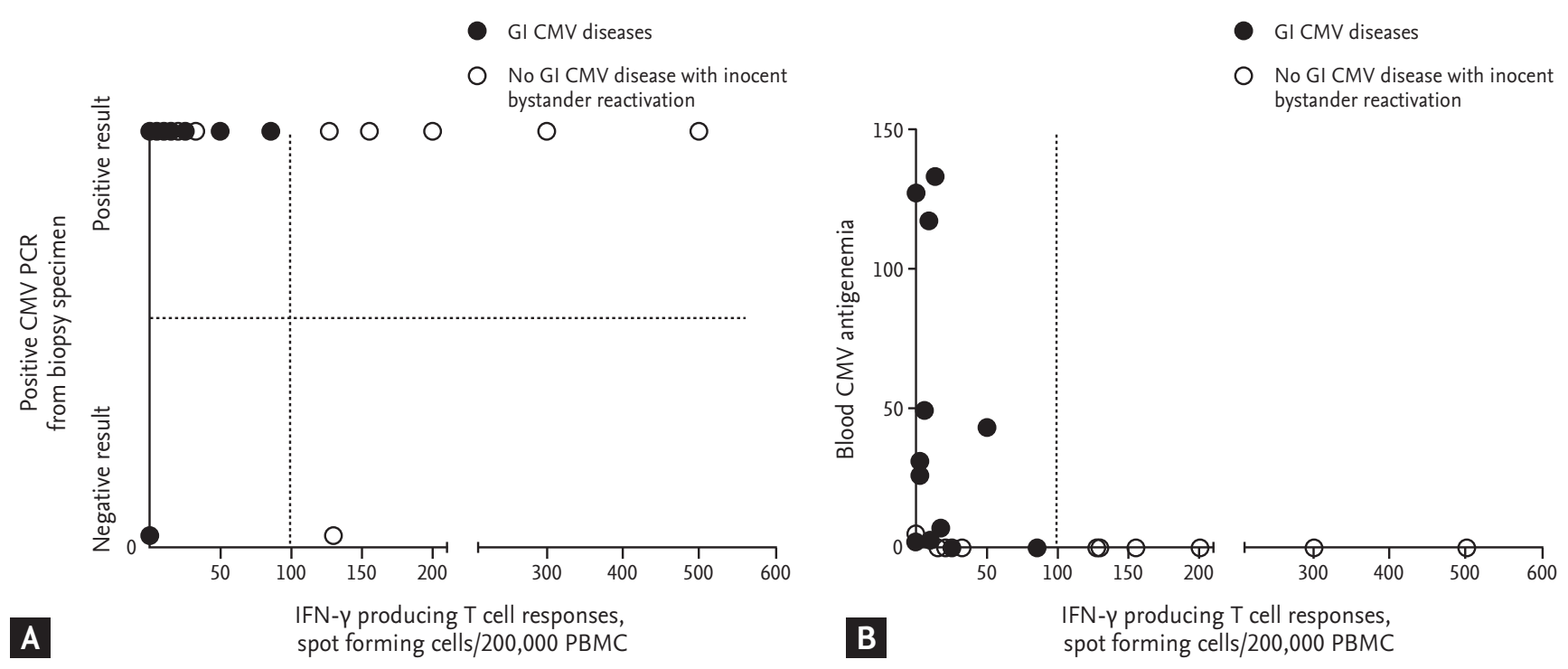

Indeterminate GI CMV diseases

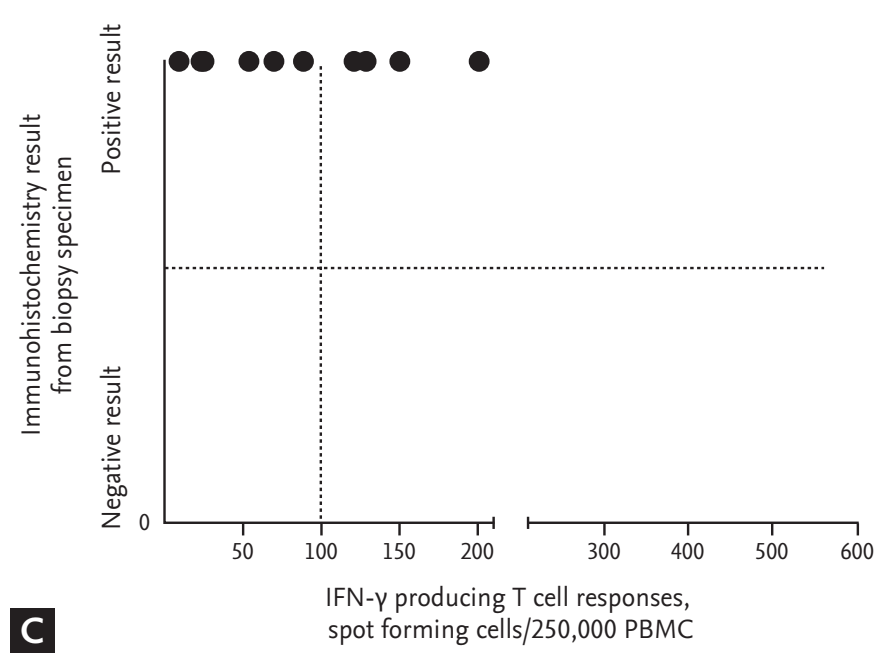

Supplementary Figure 2. The 2-axis model in which the $\mathrm{x}$-axis is the cytomegalovirus (CMV) enzyme-linked immunospot assay result and the $y$-axis the result of tissue CMV polymerase chain reaction (PCR) (A) and blood CMV antigenemia (B) in patients with suspected gastrointestinal CMV disease, and CMV immunohistochemical staining (C) in patients with indeterminate gastrointestinal CMV disease. GI, gastrointestinal; IFN, interferon; PBMC, peripheral blood mononuclear cells. 